\title{
Alcohol Detection with Vehicle Controlling
}

\author{
Dimple U. Mendhe ${ }^{1}$, Pallavi R. Mishra ${ }^{2}$, Monika R. Taywade ${ }^{3}$, Rajani G. Musale ${ }^{4}$, Komal Ramteke \\ Student, Dept of IT, RGCER, Nagpur, Maharashtra, India ${ }^{1,2,3,4}$ \\ Assistant Professor, Dept of IT, RGCER, Nagpur, Maharashtra, India ${ }^{5}$
}

\begin{abstract}
This system provides a unique method to curb drunken people. The system has an alcohol sensor embedded on the steering of the car. Whenever the driver starts ignition, the sensor measures the content of the alcohol in his breath and automatically switches off the car if he is drunken. In this system the sensor delivers a current with a linear relationship to the alcohol molecules from zero to very high concentration. The output of the sensor is fed to the pic microcontroller for comparison. If the measured value reaches the threshold, relay cut off automatically and the buzzer produces sound.
\end{abstract}

Keywords: PIC16F877A, CMOS, MQ-3 gas sensor.

\section{INTRODUCTION}

This system detects the content of alcohol in the breath and thus it attempts to clamp down alcoholics. This system uses PIC16F877A, LCD display, MQ-3gas sensor, relay and buzzer. The output of the sensor is directly proportional to the content of alcohol consumed. Nowadays alcohol sensor play a significant role in our society and it has vast applications.

This type of sensors in cars is a great safety factor which can be embedded in the steering of the cars. When the driver starts the ignition, sensor measures the content of the alcohol in his breath and automatically switches off the car which will stop the drink driving offenders. Thus we can reduce alcohol related road accidents and hence these kinds of detectors have a great relevance. It can also be used in schools, colleges, offices and some public places such as hospitals, libraries etc.

\section{A. Aim and Objective:}

The main purpose behind this project is "Drunk driving detection". Now a days, many accidents are happening because of the alcohol consumption of the driver or the person who is driving the vehicle. Thus Drunk driving is a major reason of accidents in almost all countries all over the world. Alcohol Detector in Car project is designed for the safety of the people seating inside the car.

Alcohol breath analyzer project should be fitted / installed inside the vehicle. The main unit of this project is an "Alcohol sensor". If the person inside car has consumed alcohol then it is detected by the sensor. Sensor gives this signal to a comparator IC.

The output of comparator is connected to the microcontroller. Microcontroller is the heart of this project. It is the CPU of the complete circuit. Microcontroller gives high pulse to the buzzer circuit and the buzzer is turned on. At the same time a relay is turned off. Due to this the ignition of the car is deactivated.

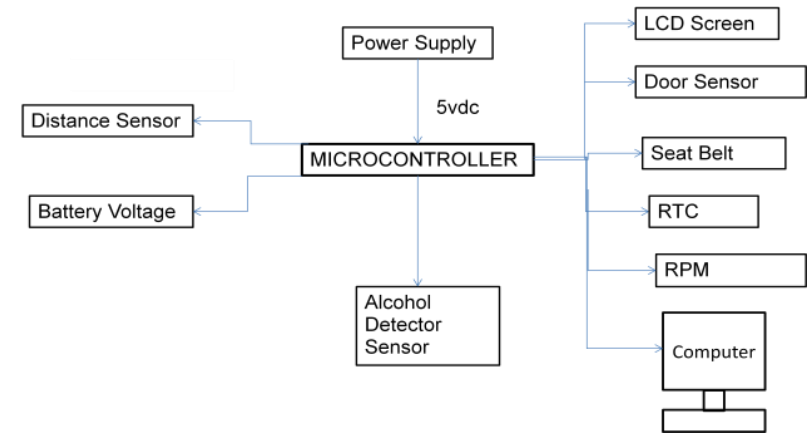

Fig.1. Block Diagram of ADVC

\section{PROPOSED WORK}

An embedded system is some combination of computer hardware and software, either fixed in capability or programmable, that is specifically designed for a particular function Since the embedded system is dedicated to specific tasks, design engineers can optimize it reducing the size and cost of the product and increasing the reliability and performance. Embedded systems are controlled by one or more main processing cores that are typically either a microcontroller or a digital signal processor (DSP). Embedded systems control many devices in common use today.

\section{A. Alcohol Sensor}

This is an alcohol sensor, which detects ethanol in the air.

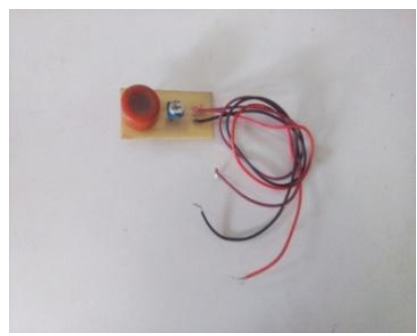

Fig.2.MQ-3 Alcohol Sensor 
It is one of the straight forward gas sensors so it works almost the same way with other gas sensors. It costs $\$ 6.90$. Typically, it is used as part of the breathalyzers or breath testers for the detection of alcohol in human breath.

\section{B. Display Unit}

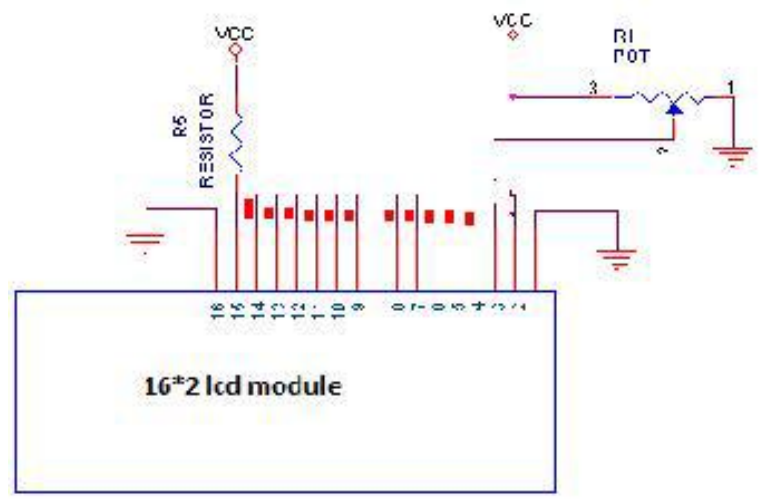

Fig.3. Liquid Crystal Display

LCD display is used for displaying the message that sent from the remote location. The LCD module is a dotmatrix liquid crystal display that displays alphanumeric, kana (Japanese characters) and symbols. The CMOS technology makes the device ideal for applications in hand-held portable and other battery-powered instruments with low power applications. Most LCDs with 1 controller has 14 Pins and LCDs with 2 controller has 16 Pins (two pins are extra in both for backlight LED connections). Above is the quite simple schematic. It consists of 16 pins ( 8 data lines, 3 control lines, 2 power lines, 1 contrast line and 2 pins for back light LED connection). Data line and control line are connected to the microcontroller. The LCD panel's Enable and Register Select is connected to the Control Port. The Control Port is an open collector / open drain output. When connecting LCD module to a parallel I/O device, the burden of ensuring proper operation falls on the software. While most Parallel Ports have internal pull-upresistors, there is a few which don't. Therefore by incorporating the two $10 \mathrm{~K}$ external pull up resistors, the circuit is more portable for a wider range of computers, some of which may have no internal pull up resistors. At the interface of LCD module, there are three power supply terminals- Vdd, GND, Vo. The LCD is driven by a voltage which is determined by Vdd-Vo. The data bus lines are DB7-DB0. When the enable signal is at the low level, this data bus terminals will remain in a high impedance state. When the data bus is open it produces a high output voltage. When the busy flag is at a high level, it indicates that the controller is in the internal operation mode and the next instruction will not be accepted. The next instruction must be written after the busy flag goes low. The delay should be suitable for most machines. If the LCD panel is not initializing properly, you can try increasing the delays. The LCD module is automatically initialized or reset when the power is turned on using the internal reset circuit. The busy flag holds 1 and does not accept instructions until initialization ends. The busy state lasts for 15 minutes after Vdd rises to 4.5 volts. When power supply restrictions are not met, the internal reset circuit will not operate normally and the initialization will not be performed. In this case, the controller should be initialized by the MPU according to "initializing by instruction".

C. Microcontroller

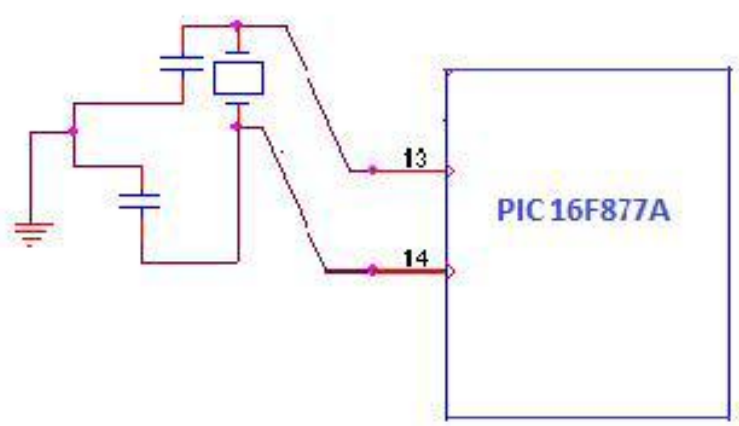

Fig.4. PIC16F877A

A microcontroller is used for controlling entire circuits and to maintain timings. Here a microcontroller named PIC16F877A from Microchip corporation is used for this purpose. It is a 16 bit microcontroller and has 40 pins arranged in $4 \mathrm{I} / \mathrm{O}$ ports, that ports are used here for connecting LCD module and to interface with the load. As shown in the above figure microcontroller is wired with a crystal for clock and each pin of crystal is connected to ground through capacitors to avoid noise pulse in the clock. Here a $4 \mathrm{MHz}$ crystal is used that gives $4 \mathrm{MHz}$ clock for the microcontroller.

D. Power supply and voltage regulator

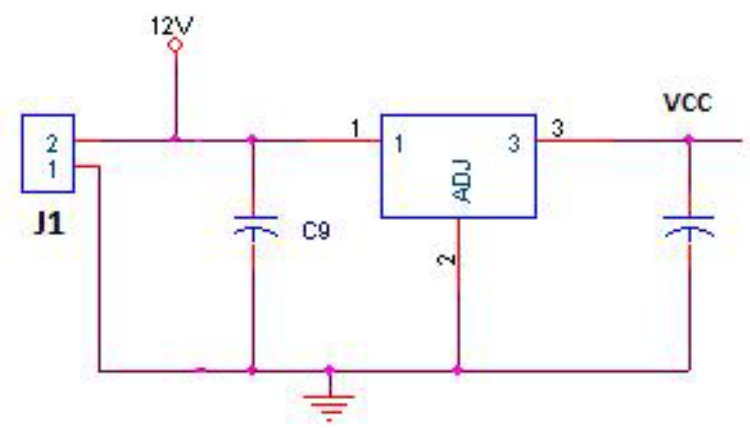

Fig.5. Power supply and voltage regulator

The circuit diagram of the power supply unit is shown above. It mainly consists of a voltage regulator (here it is 7805). The voltage regulator plays an important role in a power supply unit. Output of the power supply unit is always dc which is given to the controller. The primary purpose of the regulator is to aid the rectifier and filter circuit in providing a constant dc voltage to the device. Power supplies without regulators have an inherent problem of changing of dc voltage values due to variations 
in the load or due to fluctuations in the input voltage. With regulator connected to the dc output, the voltage can be maintained with a close tolerant region of desired output.

\section{FLASH MAGIC SOFTWARE}

Flash magic is Windows software from the Embedded Systems Academy that allows easy access to all the ISP features provided by the devices. These features include:

- Erasing the Flash memory (individual blocks or the whole device)

- Programming the Flash memory

- Modifying the Boot Vector and Status Byte

- Reading Flash memory

- Performing a blank check on a section of Flash memory

- Reading the signature bytes

- Reading and writing the security bits

Direct load of a new baud rate (high speed communications)

- Sending commands to place device in Bootloader mode Flash Magic provides a clear and simple user interface to these features and more as described in the following sections. Under Windows, only one application may have access the COM Port at any one time, preventing other applications from using the COM Port. Flash Magic only obtains access to the selected COM Port when ISP operations are being performed. This means that other applications that need to use the COM Port, such as debugging tools, may be used while Flash Magic is loaded. Note that in this manual third party Compilers are listed alphabetically. No preferences are indicated or implied.

\section{Main Window}

The following is a screenshot of the main Flash Magic window. The apperance may differ slightly depending on the device selected

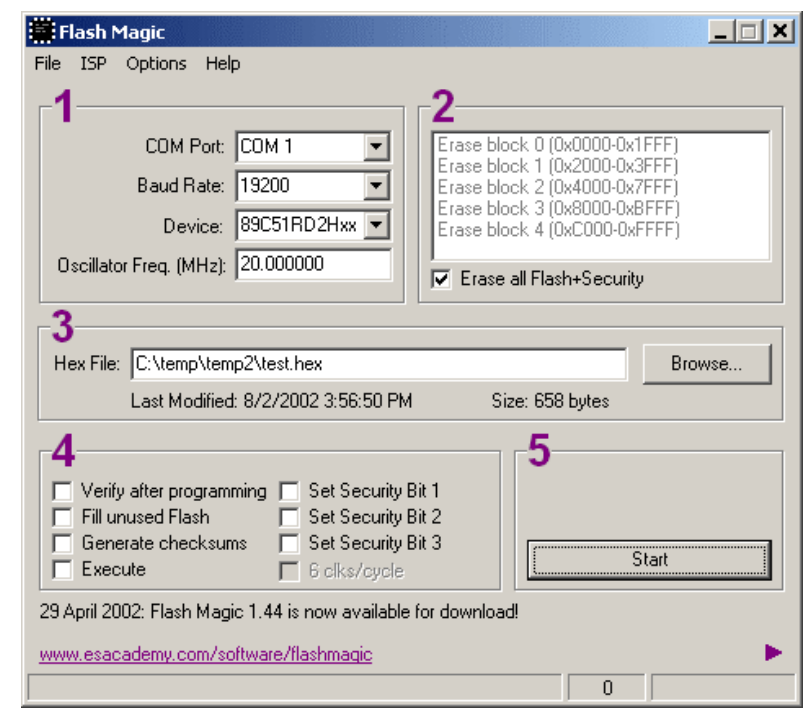

The window is divided up into five sections. Work your way from section 1 to section 5 to program a device using the most common functions. Each section is described in detail in the following sections. At the very bottom left of the window is an area where progress messages will be displayed and at the very bottom right is where the progress bar is displayed. In between the messages and the progress bar is a count of the number of times the currently selected hex file has been programmed since it was last modified or selected.

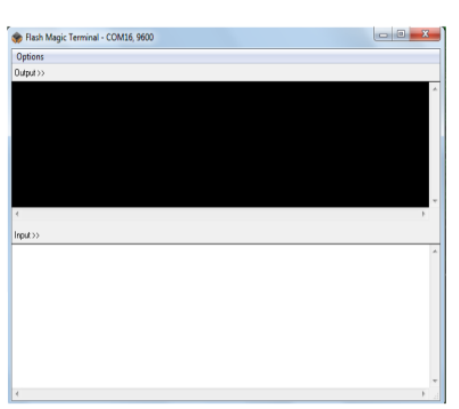

\section{RESULT AND FUTURE WORK}

The The circuit has an alcohol sensor. This sensor measures the content of alcohol from the breath of drunken people. The sensor delivers a current with linear relationship to the alcohol molecules from zero to very high concentrations. Output of the sensor is directly proportional to the alcohol content. When the alcohol molecules in the air meet the electrode that is between alumina and tin dioxide in the sensor, ethanol burns into acetic acid then more current is produced. So the more alcohol molecules more will be the current produced. Because of this current change, we get the different values from the sensor. Output of the sensor is then fed to the microcontroller for comparison. The output of the sensor is in the analog nature which should be converted into digital format. This is done by the analog to digital converter of the microcontroller unit. The microcontroller controls the entire circuit. When the measured value reaches the threshold (here it is 255) the microcontroller switches the ignition $\mathrm{ON}$. Then relay cuts off automatically and buzzer produces sound. The LCD displays the message that sent from the microcontroller unit. The working conditions and various constraints were properly studied before carrying out further steps. The components were purchased and the circuit was initially set on the breadboard. The PCB was fabricated as per the requirement and was soldered with components, taking proper care to avoid shorting between various connections. The output was verified on the PCB. The circuit worked successfully.

\section{CONCLUSION AND DISCUSSION}

Our project Alcohol Detection System was implemented successfully. This device provides much advanced facilities in now a days life as it can be easily implemented in vehicles. Thus we can reduce alcohol related road 
accidents and hence these kinds of detectors have a great relevance. It can also be used in schools, colleges, offices and some public places such as hospitals, libraries etc. Through this project we present hardware programming of microcontroller to facilitate as alcohol sensor.

\section{REFERENCES}

[1] Dr. Charles Kim,Embedded computing with pic16F877A

[2] Martin Jawitz,Printed circuit board materials hand book

[3] G. Hayes, F. Blosser, "Motor Vehicle Crashes Claim More than a Million Accident Position Lives Worldwide", CDC Injury Center Media Relations, Press Release, At The Ajkident April, 2004.

[4] http://www.airbagcrash.com (General Motor Event Data Recorders)

[5] Thomas K. Kowalick, "Black Boxes: Event Data Recorders", MICAH,summer 2005.

[6] Dr. charles Nim.Embeded computing.

[7] www.alldatabooks.com

[8] www.alldatasheets.comX-Y

[9] The 8051 Micro controller and Embedded Systems -Muhammad Ali Mazidi 